\title{
Multilayered dynamic strain gauge using of piezoelectric PVDF films
}

\author{
Nelly Rogacheva ${ }^{*}$ \\ Moscow state university of civil engineering, Yaroslavskoye shosse, 26, Moscow, Russia, 129337
}

\begin{abstract}
The strain gauge made of a piezoelectric film is proposed. The operation of the gauge is based on the piezoelectric effect: the deformation of the gauge causes an electric potential proportional to the deformation. The gauge consists of piezoelectric layers, covered by metal electrodes and adhesive layers that connect the film layers to a multilayer rectangular bar. Since the piezoelectric film has a small modulus of elasticity, the strain gauge can be used to measure the deformation of structures from soft materials.
\end{abstract}

\section{Introduction}

Analysis of stress-strain state of structures, as a rule, is performed on the basis of measuring deformations on the surface of the body under study. The strain gauge must satisfy the following requirements:

- The gauge measurements must have temporal and temperature stability;

- The gauge measurements must have good accuracy;

- The dimensions of the gauge should be small enough to correctly measure the deformation at the point of the body;

- The influence of the gauge on the stress-strain state of the body in the vicinity of the point under study should be negligible;

- The gauge should be of low cost and easy to use;

Among the experimental methods of measuring deformations, the most widely used strain gauges based on the idea of Lord Kelvin [1,2]. Kelvin found, the conductor subjected to deformation, slightly changes resistance. This small change in resistance can be measured. The strain resistance gauge measures a secondary small value - a change in resistance and requires special equipment to measure it.

The dynamic strain gauge from piezoceramics was first proposed in [3] and then in [4].The gauge is a piezoceramic rectangular thin plate with a thickness pre-polarization. The plate faces are covered with electrodes. The plate is glued to the surface of the body being examined and deformed together with it. Due to the piezoelectric effect, when the plate is deformed, an electric charge appears on its electrodes, which is easy to measure. If the piezoceramics possesses a strong piezoelectric effect, then a significant part of the mechanical deformation energy transforms into electrical energy. For example, for

* Corresponding author: nrogache@yandex.ru 
piezoceramics PZT-4, PZT-5, more than $30 \%$ of mechanical energy is converted into electrical energy.

The elastic compliance of piezoceramics is a quantity of the same order as the elastic compliance of aluminum. Such a gauge will distort the stress-strain state at the studied point of the deformable body from a soft material with a small modulus of elasticity.

\section{Basic equations and boundary conditions}

Consider a piezoelectric rectangular bar. Its length is $2 l$, the width is $2 m$, and the thickness is $2 h$. We assume that the length of the bar is much larger than its width and the thickness: $l>>m$ and $l>>h$. The bar consists of $n$ layers of piezoelectric film. Each layer is prepolarized to the thickness direction and it faces covered by electrodes. The layers of the piezoelectric film are glued together. One of external faces is glued to the investigated body with an insulating adhesive.

We place the piezoelectric bar in a Cartesian coordinate system: the $\mathrm{x}_{1}$ axis is directed along the length of the bar, the $\mathrm{x}_{2}$ axis is directed along the width of the bar, the $\mathrm{x}_{3}$ axis is perpendicular to the plane $\mathrm{x}_{1} \mathrm{x}_{2}$ and is directed along the thickness of the bar: $-l \leq x_{1} \leq+l$, $m \leq x_{2} \leq+m,-h \leq x_{3} \leq+h$. The gauge consists of repeating sets of three layers: an adhesive layer, an metal layer (electrode), a PVDF film layer. Since any set of three layers has the same stress-strain state, we will consider only one set to be called the composite layer. The thicknesses of the PVDF layer, the electrode, and the adhesive layer are $2 h_{f}, 2 h_{m}$, and $2 h_{a}$ respectively.

We write out only those equations which we will need later:

The constitutive relations for elastic layers (Hooke's law)

$$
\sigma_{i, c}=\frac{E_{c}}{1-v_{c}^{2}}\left(e_{i}+v_{c} e_{j}\right)+\frac{v_{c}}{1-v_{c}} \sigma_{3, c}
$$

The index $c$ should be replaced by $a$ for the adhesive layer and by $m$ for the electrode. Piezoeffect equations

$$
\begin{gathered}
\sigma_{i}=\frac{1}{s_{11}^{E}\left(1-v^{2}\right)}\left(e_{i}+v e_{j}\right)-\frac{s_{13}^{E}}{s_{11}^{E}(1-v)} \sigma_{3}-\frac{d_{3 i}+v d_{3 j}}{s_{11}^{E}\left(1-v^{2}\right)} E_{3} \\
D_{3}=\varepsilon_{33}^{T} E_{3}+d_{31} \sigma_{1}+d_{32} \sigma_{2}+d_{33} \sigma_{3}
\end{gathered}
$$

Strain-displacement formula

$$
e_{i}=\frac{\partial u_{i}}{\partial x_{i}}
$$

Electrostatic equation

$$
E_{3}=-\frac{\partial \psi}{\partial x_{3}}
$$

Here $u_{1}$ and $e_{1}$ are the displacement $u_{1}$ and strain $e_{1}$ in the $x_{1}$ direction, $E_{3}$ and $D_{3}$ are the components of the electric field vector and electric induction vector in the $x_{3}$ direction, $s_{11}^{E}$ is the elastic compliance at constant electric field, $d_{31}$ and $d_{32}$ are the piezoelectric 
constants, $\varepsilon_{33}^{T}$ is the permittivity for zero stresses, $\psi$ is the electric potential. The notation in use coincides with taken previously [5]. Every equations with subscripts $i$ and $j$ contains two equations: one for $i=1$ and $j=2$ and the other for $i=2$ and $j=1$.

We assume that on the contact area $x_{3}=-h$ of the gauge and the body the displacements of the bar are $u_{i}, u_{3}$ equal to the displacement of the studied body $v_{i}, v_{3}$ :

$$
x_{3}=-h: v_{i}=u_{i}, \quad v_{3}=u_{3}
$$

On the remaining faces of the bar we have the conditions:

$$
\begin{gathered}
x_{3}=+h: \sigma_{i 3}=\sigma_{3}=0 \\
x_{1}= \pm l: D_{1}=0, \quad \sigma_{11}=\sigma_{13}=\sigma_{12} \\
x_{2}= \pm m: D_{2}=0, \sigma_{21}=\sigma_{23}=\sigma_{22}
\end{gathered}
$$

The electrical potential on the electrodes of each layer is known (measured):

$$
\left.\psi\right|_{x_{3}=h_{a}}=-V,\left.\quad \psi\right|_{x_{3}=h_{a}+2 h_{f}}=V
$$

Since the electrodes are connected by an external contour, the integral condition should hold

$$
\int_{s}^{\bullet} \dot{D}_{3} d s=2 V Y
$$

Where the integral is taken over the surface $s$ of one of the electrodes and the point denotes the time derivative.

For simplicity we will assume that the body and the gauge vibrate by the law $e^{-i \omega t}$

$$
-i \omega \int_{s} D_{3} d s=2 V Y
$$

where $\omega$ is the angular frequency of vibrations and $t$ is the time. Since the vibrations are harmonic, we will describe them by equations written for the amplitude values of the needed quantities

\section{One-dimensional strain gauge}

Piezoelectric film with greatly different piezoelectric constants is an ideal material for a one-dimensional gauge. For example, for Kureha KF piezoelectric film PVDM MDMs the piezoelectric constants $d_{31}$ is much larger than $d_{32}$ [6]:

$$
d_{31}=2.17 \cdot 10^{-11} \mathrm{~m} / \mathrm{V}, d_{32}==1.87 \cdot 10^{-12} \mathrm{~m} / \mathrm{V}
$$

The measured voltage is proportional to the value $d_{31}$.

The gauge theory is based on the hypotheses similar to those proposed for piezoceramic gauge [5]. 
First hypothesis

We assume that the dimensions of the gauge are small enough to treat deformations in the contact area of the gauge and the body under investigation as constant.

Second hypothesis

In the equations $(1)-(3)$ the stresses $\sigma_{2}$ and $\sigma_{3}$ can be neglected as compared to the principal stress $\sigma_{1}$. Using this hypothesis, we rewrite the equations in a simplified form: for the elastic layers

$$
\sigma_{1 c}=\frac{E_{c}}{1-v_{c}^{2}}\left(e_{1}+v_{c} e_{2}\right), \quad e_{2}+v_{c} e_{1}=0
$$

for the piezoelectric layers

$$
\begin{gathered}
\sigma_{1}=\frac{1}{s_{11}^{E}\left(1-v^{2}\right)}\left(e_{1}+v e_{2}\right)-\frac{d_{31}+v d_{32}}{s_{11}^{E}\left(1-v^{2}\right)} E_{3} \\
\frac{1}{s_{11}^{E}\left(1-v^{2}\right)}\left(e_{2}+v e_{1}\right)-\frac{d_{32}+v d_{31}}{s_{11}^{E}\left(1-v^{2}\right)} E_{3}=0 \\
D_{3}=\varepsilon_{33}^{T} E_{3}+d_{31} \sigma_{1}
\end{gathered}
$$

We find the strain $e_{1}$ from the second equation (10) and substitute it in the first equation, as a result we obtain

$$
\sigma_{1, c}=E_{c} e_{1}
$$

Similarly we transform the equations (11) for $\mathrm{f}$ the piezoelectric layers

$$
\begin{gathered}
\sigma_{1}=\frac{e_{1}}{s_{11}^{E}}-\frac{d_{31}}{s_{11}^{E}} E_{3} \\
D_{3}=\varepsilon_{33}^{T}\left(1-k_{31}^{2}\right) E_{3}+\frac{d_{31}}{s_{11}^{E}} e_{1}
\end{gathered}
$$

Third hypothesis

We assume that the electrical potential varies with the thickness by the linear law

$$
\psi=\psi,{ }_{0}+x_{3} \psi, 1
$$

Hence, using the relation (5) and the conditions (8), we get

$$
E_{3}=-\frac{V}{h}
$$

Since the components of the strain $e_{1}$ and the strength of the electric field $E_{3}$ are constant over the whole area of the gauge, from Eq. (13) follows that the component $D_{3}^{(k)}$ (14) of the electrical induction vector is constant too. Then the equation for a closed electric circuit (9) after integration over the surface of one electrode will take the form

$$
-i \omega s D_{3}=2 V Y
$$

We solve this equations according to the deformation $e_{1}$ taking into account the relations (14), (15) 


$$
e_{1}=i \frac{2 V Y}{\omega s} \frac{s_{11}^{E}}{d_{31}}+d_{31} \frac{1-k_{31}^{2}}{k_{31}^{2}} \frac{V}{h_{f}}
$$

where s equals $2 l \times 2 m$.

From the formula (16) the strain $e_{1}$ for harmonic vibrations is calculated using the measured electrical potential difference.

To increase the measured electrical potential difference, we should connect the electrodes on the surfaces of the piezoelectric film layers in series. If the gauge consists of $n$ composite layers, the electrical potential will increase $n$ times.

In the constructed theory of the 1D gauge the boundary conditions (7), (9) - (11) are satisfied approximately. The complete error is defined by the formula [5] $\varepsilon=O(m / l, h / l)$.

\section{Electromechanical coupling coefficient}

An important characteristic of the performance of piezoelectric elements is the electromechanical coupling coefficient (EMCC). The EMCC is commonly used as a measure of a piezoelectric element's efficiency. It gives the ratio of the electrical (mechanical) energy stored in the volume of the piezoelectric body and capable of conversion to the total mechanical (electrical) energy supplied to the body. In $[5,7]$ it was shown that the energy formula the EMCC is the universal formula. The energy formula for computing the EMCC $k_{e}$ has the form

$$
k_{e}^{2}=\frac{U^{(d)}-U^{(s h)}}{U^{(d)}}
$$

where $U^{(d)}$ is the internal energy of the body when the electrodes are disconnected and $U^{(s h)}$ is the internal energy for short-circuited electrodes.

In order to find $U^{(d)}$ and $U^{(s h)}$ we should solve first our initial problem and then to solve two additional problems. In solving the additional problems, we suppose that

$$
e_{1}^{(d)}=e_{1}^{(s h)}=e_{1}
$$

where $e_{1}$ is the component of strain found from the solution of the initial problems.

We find the quantity $U^{(d)}$ using the condition for disconnected electrodes

$$
D_{3}^{(d)}=\varepsilon_{33}^{T}\left(1-k_{31}^{2}\right) E_{3}^{(d)}+\frac{d_{31}}{s_{11}^{E}} e_{1}=0
$$

From this equation we obtain

$$
E_{3}^{(d)}=-\frac{k_{31}^{2}}{1-k_{31}^{2}} \frac{e_{1}}{d_{31}}, \quad \sigma_{1}^{(d)}=\frac{1}{1-k_{31}^{2}} \frac{e_{1}}{s_{11}^{E}}
$$

To determine $U^{(s h)}$ we use the conditions for short-circuited electrodes

$$
\left.\psi^{(s h)}\right|_{s}=0
$$

Hence it follows 


$$
E_{3}^{(s h)}=0, \quad \sigma_{1}^{(s h)}=\frac{e_{1}}{s_{11}^{E}}
$$

Taking into account the energy of the elastic layers we write the formulas for the quantities $U^{(d)}$ and $U^{(s h)}$

$$
\begin{aligned}
& U^{(d)}=\int_{v}\left(\sigma_{1}^{(d)} e_{1}+E_{3}^{(d)} D_{3}^{(d)}+\sigma_{1 m} e_{1}+\sigma_{1 a} e_{1}\right) d v \\
& U^{(s h)}=\int_{v}\left(\sigma_{1}^{(s h)} e_{1}+E_{3}^{(s h)} D_{3}^{(s h)}+\sigma_{1 m} e_{1}+\sigma_{1 a} e_{1}\right) d v
\end{aligned}
$$

After integrating by the volume $v$ and simple transformations we get

$$
\begin{gathered}
U^{(d)}=2 s e_{1}^{2}\left(\frac{h_{f}}{s_{11}^{E}\left(1-k_{31}^{2}\right)}+h_{m} s_{11}^{E} E_{m}+h_{a} s_{11}^{E} E_{a}\right) \\
U^{(s h)}=2 s e_{1}^{2}\left(\frac{h_{f}}{s_{11}^{E}}+h_{m} s_{11}^{E} E_{m}+h_{a} s_{11}^{E} E_{a}\right)
\end{gathered}
$$

We substitute the quantities $U^{(d)}$ and $U^{(s h)}$ into the formula (17) and find $k_{e}^{2}$

$$
k_{e}^{2}=\frac{k_{31}^{2}}{\left(1-k_{31}^{2}\right)\left(1+s_{11}^{E} E_{m} h_{m} / h_{f}+s_{11}^{E} E_{a} h_{a} / h_{f}\right)}
$$

This formula can be simplified: the value $k_{31}^{2}$ is a negligibly small quantity as compared with 1 . The $k_{31}$ is of the order 0.02 .

$$
k_{e}^{2}=\frac{k_{31}^{2}}{\left(1+s_{11}^{E} E_{m} h_{m} / h_{f}+s_{11}^{E} E_{a} h_{a} / h_{f}\right)}
$$

\section{Conclusions}

The advantage of the PVDF film gauge are the following:

- the gauge is simple in structure and easy to use;

- the gauge can be made of any size;

- $\quad$ the gauge is suitable for soft structures.

\section{References}

1. W. Thomson (Lord Kelvin), Proc. R. Soc.,(London, 1856)

2. A. S. Kobayashi, ed., Handbook on experimental mechanics (Prentice-Hall, New Jersey, 1987)

3. A. A. Infimovskaya, G. N. Chernyshev, N. N. Rogacheva, Mech. of Solids, 24, 2 (1989)

4. H. S. Tzou, Piezoelectric Shells (Kluver, Dordrecht, 1993) 
5. N. N. Rogacheva, The theory of piezoelectric shells and plates (CRC Press, Roca Raton, 1994)

6. T. Sato, H. Ishikawa, O. Ikeda, Appl. Optics, 21, 20 (1982)

7. C. C. Chou, N. N. Rogacheva, S. H. Chang, IEEE Trans. Ultr., Ferr. and Freq. contr., 42, 4 (1995) 